\title{
The S-Matrix in Classical Mechanics
}

\author{
W. HUNZIKER \\ Institut des Hautes Etudes Scientifiques, Bures-sur-Yvette
}

Received November 4, 1967

\begin{abstract}
In the Hilbert-space version of classical mechanics, scattering theory for $N$-particle systems is developed in close analogy to the quantum case. Asymptotic completeness is proved for forces of finite range. Infinite-range forces lead to the problem of stability of bound states and can be dealt with only in some simple cases.
\end{abstract}

\section{Introduction}

For obvious reasons, collision phenomena have been studied mostly in the framework of quantum mechanics. Our aim is to show that the concepts and even some mathematical methods of the quantum theory of scattering are equally applicable to classical $N$-particle systems [1].

To make the analogy as close as possible, we work with the Hilbertspace formulation of classical mechanics. Using a suitable definition of bound states, time-dependent scattering theory is then simply copied from the quantum case. For forces of finite range, it is shown that bound states and scattering states span the entire Hilbert-space (asymptotic completeness), which implies that the $S$-matrix is unitary.

Compared with the quantum case, the restricions we put on the (two-body-) forces are much more severe. This is inevitable since already the existence of solutions to the equations of motion is established only for a smaller class of interactions. And in scattering theory again, we have to impose stronger conditions on the behaviour of the forces at infinity: in general we can construct scattering states (or the waveoperators) only if the forces are strictly zero beyond a finite range. The obstacle here is the lack of information on the stability of multiparticle bound states. In some cases where this problem does not arise or can be handled (i.e. for the channels in which only free particles or two-body bound states are present for $t \rightarrow \pm \infty$ ), infinite-range force decreasing faster than the Coulomb-force are admitted.

It is a pleasure to thank Prof. L. Motchane for his kind hospitality at the I.H.E.S., where most of this work was done, and where the author profited from discussions with D. RUELLE and O. E. LANFORD. 


\section{General Remarks on Dynamics}

We consider a system of $N$ particles interacting via two-body forces, characterized by the Hamiltonian

$$
H=\sum_{i=1}^{N} \frac{p_{i}^{2}}{2 m_{i}}+\sum_{i<k} V_{i k}\left(x_{i}-x_{k}\right)=H_{0}+V .
$$

Its states are taken as elements of the Hilbert-space $\mathscr{H}=L^{2}(\Gamma)$, $\Gamma=$ phase space. The evolution in time of a state $\psi$ is then to be determined from the equation of motion

$$
\frac{d \psi}{d t}=\{H, \psi\} \equiv L \psi
$$

where $\{$,$\} is the Poisson-bracket, defining the Liouville-operator L$. Explicitly,

$$
\begin{aligned}
L & =L_{0}+L_{V} \\
L_{0}=-\sum_{i=1}^{N} \frac{p_{i}}{m_{i}} \frac{\partial}{\partial x_{i}}, L_{V} & =\sum_{i<k} \operatorname{grad} V_{i k}\left(\frac{\partial}{\partial p_{i}}-\frac{\partial}{\partial p_{k}}\right) .
\end{aligned}
$$

If the forces are locally square-integrable, $L_{0}$ and $L_{V}$ are well-defined and skew-symmetric on the dense domain $C_{0}^{1}(\Gamma)$. It is less trivial to decide whether $L$ has a (preferably unique) skew-adjoint extension which then generates a one-parameter unitary group describing the dynamics of the system. The analogous question in quantum mechanics has a positive answer for a large class of interactions, for example if (the operator) $V$ is relatively small with respect to $H_{0}$ (i.e. if $\|V \psi\| \leqq a\left\|H_{0} \psi\right\|+b\|\psi\|$ for some $a<1, b<\infty$ and all $\psi \in D\left(H_{0}\right)$. Then $H_{0}+V$ is selfadjoint [2]). This kind of argument fails in the classical case, since $L_{V}$ cannot possibly be estimated in terms of $L_{0}$, regardless of how wellbehaved $V$ is ${ }^{1}$. To define the dynamics of the system, we better start from the canonical equations

$$
z_{i}=\varepsilon_{i k} \frac{\partial H}{\partial z_{k}},
$$

where $\left(z_{1} \ldots z_{6 N}\right)=\left(x_{1} \ldots x_{N}, p_{1} \ldots p_{N}\right)$ and where $\varepsilon$ is the matrix of the transformation $\left(x_{i}, p_{i}\right) \rightarrow\left(p_{i},-x_{i}\right)$. Throughout this paper we shall assume that the potentials satisfy the following conditions:

(A) $V_{i k}(x)$ is a continuous function $R^{3} \rightarrow[b,+\infty]$ with $b>-\infty$.

(B) For any $W<\infty, V_{i k}(x)$ is twice continuously differentiable, with uniformly bounded derivatives, in the region $\left\{x: V_{i 1 c}(x)<W\right\}$.

The phase space of the system is then the open subset

$$
\Gamma=\{z: H(z)<\infty\}
$$

1 This is also the reason why the resolvent equations, which form the basis of time-independent scattering theory in quantum mechanics, are not likely to be useful in the classical case. 
of $R^{6 N}$, and the right-hand side of (2) is uniformly Lipshitz (in the sense of the Euclidean norm on $R^{6 N}$ ) in any region of bounded energy. Since energy is conserved, we conclude that (2) has a unique global solution $z\left(t, z_{0}\right)$ for any initial value $z_{0} \in \Gamma$. Furthermore, $z\left(t, z_{0}\right)$ is once continuously differentiable with respect to $z_{0}$ and the derivatives satisfy the familiar exponential estimate

$$
\left|\frac{d z}{d z_{0}}\right| \leqq e^{K|t|},
$$

where the left-hand side is the norm of the matrix $\partial z_{i} / \partial z_{0 k}$, considered as an operator on the Euclidean space $R^{6 N}$, and where $K$ is a Lipshitzconstant for the region $\left\{z: H(z)<H\left(z_{0}\right)+1\right\}$. In fact, the mappings

$$
S^{t}: z_{0} \rightarrow z\left(t, z_{0}\right), \quad-\infty<t<+\infty
$$

form a one-parameter group of canonical transformations of $\Gamma$ onto $\Gamma$, which in turn induces a strongly continuous unitary group $\exp (L t)$ on $L^{2}(\Gamma)$ by

$$
\left(e^{L t} \psi\right)(z)=\psi\left(S^{-t} z\right) .
$$

The Liouville-operator $L$ is now defined as the generator of this group. Then we have

Lemma 1. (a) $C_{0}^{1}(\Gamma)$ is invariant under the group $e^{L t}$.

(b) $C_{0}^{1}(\Gamma) \subset D(L)$ and on $C_{0}^{1}(\Gamma), L$ is given by $(1)$.

(c) For any $\psi \in C_{0}^{1}(\Gamma)$ there exists a constant $K<\infty$ such that

$$
\left\|e^{L t} \psi\right\|_{D} \leqq e^{K|t|}\|\psi\|_{D},
$$

where, for any $\varphi \in C_{0}^{1}(\Gamma)$, we define

$$
\|\varphi\|_{D}^{2}=\sum_{i=1}^{6 N}\left\|\frac{\partial \varphi}{\partial z_{i}}\right\|^{2}
$$

We remark that, by (a), (b) and by a lemma due to NeLson [3], we can now give an affirmative answer to the original problem: if $(\mathrm{A}, \mathrm{B})$ are satisfied, then $L$ is the closure of the operator defined by (1) on $C_{0}^{1}(\Gamma)$. NeLson's lemma: on a Banach-space $X$, let $P^{t}$ be a strongly continuous semigroup with generator $A$. Then $E \subset D(A), \bar{E}=X$ and $P^{t} E \subset E$ for all $t>0$ imply that $A$ is the closure of its restriction to $E$.

For the rest of this paper we adopt the following conventions: first, we fix the CM (= center-of-mass) of the $N$-particle system, so that $\Gamma$ reduces to the invariant subset characterized by

$$
\sum_{i=1}^{N} m_{i} x_{i}=0, \sum_{i=1}^{N} p_{i}=0
$$

which will again be denoted by $\Gamma$. This requires no changes in the foregoing analysis. Secondly, we assume the potentials to be bounded. This is not a real restriction, since we can always confine the discussion to states of bounded energy. On the other hand, we then have $T=R^{6(N-1)}$, independent of $V$, which simplifies the notation. 


\section{Bound States and Scattering States}

Clearly, the concept of bound states must be linked to boundedness of orbits in configuration space $\left\{\left(x_{1} \ldots x_{N}\right): \sum m_{i} x_{i}=0\right\}$. To describe this, any norm in this space may serve. We choose

and define:

$$
N(z)=\left(\frac{1}{2} \sum m_{i} x_{i}^{2}\right)^{1 / 2}
$$

$$
\begin{aligned}
\bar{N}(z) & =\sup _{-\infty<t<+\infty} N\left(S^{t} z\right), \\
B^{n} & =\{z: \bar{N}(z) \leqq n\}, \quad n=1,2, \ldots
\end{aligned}
$$

Since $N\left(S^{t} z\right)$ is continuous in $z, B^{n}$ is closed, therefore

$$
B=\bigcup_{n=1}^{\infty} B^{n}=\{z: \bar{N}(z)<\infty\}
$$

is measurable. The bound states are now defined as the elements of

$$
\mathscr{H}^{B} \equiv L^{2}(B) \subset \mathscr{H} .
$$

The elements of the spaces $L^{2}\left(B^{n}\right)$ are called compact bound states. They are dense in $\mathscr{H}^{B}$ since $B=\cup B^{n}$ and $B^{n} \subset B^{m}$ for $n \leqq m$ implies

$$
L^{2}(B)=\overline{\bigcup_{n=1}^{\infty} L^{2}\left(B^{n}\right)} \text {. }
$$

It is clear that $B^{n}$ and $B$ are invariant under the group $S^{t}$, hence $L^{2}\left(B^{n}\right)$ and $L^{2}(B)$ are invariant under $\exp (L t)$. In contrast to quantum mechanics, however, the part of $L$ in $\mathscr{H}^{B}$ generally has a continuous spectrum (already in the case $N=2$ ), but this is of no consequence in the following 2 .

We now use the terminology of the quantum theory of scattering [5] to define scattering states. A "channel", $\alpha$, is a partition of the set of particles $(1 \ldots N)$ into subsets $F_{1} \ldots F_{n}$ (called the "fragments" in channel $\alpha$ ), subject to the condition that each $F_{k}$ is either a single particle or a composite subsystem possessing nontrivial bound states. The "channel Hamiltonian" $H_{\alpha}$ is obtained from $H$ by dropping all interactions between different fragments, it generates a Liouville-operator $L_{\alpha}$. A motion of non-interacting, bound fragments is then described by $\exp \left(L_{\alpha} t\right) \psi_{\alpha}$, with

$$
\psi_{\alpha}(z)=\phi\left(X_{1} \ldots X_{n}, P_{1} \ldots P_{n}\right) \prod_{k=1}^{n} \varphi_{k}\left(z_{k}\right),
$$

${ }^{2}$ In fact, one should define quantum mechanical bound states similarly by a suitable condition of "boundedness" in configuration space. For nonrelativistic multiparticle systems, the discreteness of the spectrum of $H$ in $\mathscr{H}^{B}$ is then a consequence of the special form of $H[4]$, but this need not be so in other theories. 20 Commun. math. Phys., Vol. 8 
where $X_{k}=$ coordinates of the CM of $F_{k}, P_{k}=$ total momentum of $F_{k}, z_{k}=$ coordinates and momenta of the particles forming $F_{k}$ (in the CM-system of $\left.F_{k_{k}}\right), \phi \in L^{2}\left(\Gamma_{\alpha}\right)$ where $\Gamma_{\alpha}$ is the phase space of $n$ particles with masses $M_{1} \ldots M_{n}\left(M_{k}=\right.$ mass of $\left.F_{k}\right)$ with fixed $\mathrm{CM}$, and $\varphi_{k} \in \mathscr{H}_{k}^{B}$ $=$ space of bound states of the (isolated) system $F_{k}$. We define $\phi=1$ if $n=1$ and $\varphi_{k}=1$ if $F_{k}$ is a single particle. The states $\psi_{\alpha}$ span a subspace $D_{\alpha} \subset \mathscr{H}$ which may be written as

$$
D_{\alpha}=L^{2}\left(\delta_{\alpha}\right), \quad \delta_{\alpha}=\Gamma_{\alpha} \otimes B_{1} \otimes \cdots \otimes B_{n},
$$

where $B_{k}$ is the bound-state region in the phase space of $F_{k}$. Since $\delta_{\alpha}$ is invariant under the group $S_{\alpha}^{t}$ generated by $H_{\alpha}, D_{\alpha}$ is invariant under $\exp \left(L_{\alpha} t\right)$. In fact, for a state $\psi_{\alpha}$ of type (7) we have

$$
\left(e^{L_{\alpha} t} \psi_{\alpha}\right)(z)=\phi\left(X_{1}-\frac{P_{1}}{M_{1}} t, \ldots, X_{n}-\frac{P_{n}}{M_{n}} t, P_{1} \ldots P_{n}\right) \prod_{k=1}^{n}\left(e^{l_{k} t} \varphi_{k}\right)\left(z_{k}\right),
$$

where $l_{k}=$ Liouville-operator describing the internal motion of $F_{k}$. An ingoing or outgoing scattering state $\psi_{\alpha}^{ \pm}$in channel $\alpha$ is defined by the requirement that $\exp (L t) \psi_{\alpha}^{\mp} \rightarrow \exp \left(L_{\alpha} t\right) \psi_{\alpha}$ as $t \rightarrow \mp \infty$, with $\psi_{\alpha} \in D_{\alpha}$. To assert the existence of scattering states, we now assume

(C) There exists $a<\infty$ such that $V_{i k}(x)=0$ if $|x|>a$.

Then we have

Theorem 1. Under the hypothesis $(\mathrm{A}, \mathrm{B}, \mathrm{C})$,

exists for all $\psi_{\alpha} \in D_{\alpha}$.

$$
\psi_{\alpha}^{ \pm}=\lim _{t \rightarrow \pm \infty} e^{-L t} e^{L} \alpha^{t} \psi_{\alpha}
$$

Corollary. The mappings $\Omega_{\alpha}^{ \pm}: \psi_{\alpha} \rightarrow \psi_{\alpha}^{ \pm}$("wave-operators") are isometric from $D_{\alpha}$ into $\mathscr{H}$ and satisfy

$$
e^{L t} \Omega_{\alpha}^{ \pm}=\Omega_{\alpha}^{ \pm} e^{L_{\alpha} t} .
$$

Hence the ranges $R_{\alpha}^{ \pm}$of $\Omega_{\alpha}^{ \pm}$reduce the group $\exp (L t)$ and the parts of $L$ in $R_{\alpha}^{ \pm}$are unitarily equivalent to the part of $L_{\alpha}$ in $D_{\alpha}$.

Proof. It suffices to prove convergence on a dense set in $D_{\alpha}$. Such a set is spanned by the states of type (7) with the additional properties

a) $\phi\left(X_{1} \ldots X_{n}, P_{1} \ldots P_{n}\right)=F\left(X_{1} \ldots X_{n}\right) G\left(P_{1} \ldots P_{n}\right)$, with $F$ and $G$ of compact support, and supp $G$ such that it does not intersect the planes

$$
\frac{P_{i}}{M_{i}}=\frac{P_{k}}{M_{k}}(i \neq k) \text {, }
$$

b) $\varphi_{k}=$ compact bound state of $F_{k}$.

Then there exist constants $R<\infty, \sigma>0$, depending on $F, G, \varphi_{1} \ldots \varphi_{n}$, such that $z \in \operatorname{supp} \exp \left(L_{\alpha} t\right) \psi_{\alpha}$ implies

$$
\begin{aligned}
& \left|X_{i}-\frac{P_{i}}{M_{i}} t-X_{k}+\frac{P_{k}}{M_{k}} t\right| \leqq R, \\
& \left|X_{i}-x_{j}\right| \leqq R, \\
& \left|\frac{P_{i}}{M_{i}}-\frac{P_{i}}{M_{k}}\right| \geqq \sigma
\end{aligned}
$$


for all $i \neq k$, where $j$ is any particle in $F_{i}$. Let $l$ be a particle in $F_{k}, k \neq i$. Then (9) implies

$$
\left|x_{i}-x_{l}\right| \geqq \sigma|t|-3 R,
$$

hence $\operatorname{supp} \exp \left(L_{\alpha} t\right) \psi_{\alpha} \subset\left\{z: H(z)=H_{\alpha}(z)\right\}$ for $|t| \geqq T \equiv(a+3 R) / \sigma$. Consequently,

$$
e^{L}{ }_{\alpha}^{t} \psi_{\alpha}=e^{L(t \mp T)} e^{ \pm L_{\alpha} T} \psi_{\alpha}
$$

for $\pm t \geqq T$, which shows that the limits $\psi_{\alpha}^{ \pm}$exist trivially, in the sense that they are already attained at the finite times $\pm T$.

The second ingredient for the construction of the $S$-matrix is the orthogonality of scattering states in different channels:

Theorem 2. If the assertion of theorem 1 holds, then

$$
R_{\alpha}^{ \pm} \perp R_{\beta}^{ \pm} \quad(\alpha \neq \beta) .
$$

Proof. It is enough to show that

$$
\lim _{|t| \rightarrow \infty}\left(e^{L_{\alpha} t} \psi_{\alpha}, e^{L_{\beta} t} \psi_{\beta}\right)=0
$$

for $\alpha \neq \beta$ and for $\psi_{\alpha}\left(\psi_{\beta}\right)$ in the dense subset of $D_{\alpha}\left(D_{\beta}\right)$ used in the proof of theorem 1. Now $\alpha \neq \beta$ implies that there are two particles $j, l$ which belong to the same fragment in one channel (say $\alpha$ ) but to different ones in the other. The inequalities (9) (10) then show that

$$
\begin{array}{ll}
\left|x_{j}-x_{l}\right| \leqq 2 R & \text { if } \quad z \in \operatorname{supp} e^{L_{\alpha} t} \psi_{\alpha}, \\
\left|x_{j}-x_{l}\right| \geqq \sigma|t|-3 R & \text { if } \quad z \in \operatorname{supp} e^{L_{\beta} t} \psi_{\beta} .
\end{array}
$$

Therefore, the two supports do not intersect for $|t|>5 R / \sigma$, which proves (11).

By theorems 1 and 2 we can now construct the $S$-matrix as in quantum mechanics [5]. We introduce

$$
\mathscr{H}^{\prime}=\bigoplus_{\alpha} D_{\alpha}
$$

which in general cannot be viewed as a subspace of $\mathscr{H}$, since as subspaces of $\mathscr{H}$ the $D_{\alpha}$ are not mutually orthogonal. Then we define two operators $\Omega^{ \pm}$from $\mathscr{H}^{\prime}$ into $\mathscr{H}$ by

$$
\Omega^{ \pm} \Psi=\sum_{\alpha} \Omega_{\alpha}^{ \pm} \psi_{\alpha}
$$

where $\psi_{\alpha}$ is the component in $D_{\alpha}$ of $\Psi \in \mathscr{H}^{\prime}$. By virtue of theorems 1 and $2, \Omega^{ \pm}$is isometric, with range

$$
R^{ \pm}=\bigoplus_{\alpha} R_{\alpha}^{ \pm}
$$

The adjoint $\Omega^{ \pm *}$ then maps $\mathscr{H}$ onto $\mathscr{H}^{\prime}(\mathrm{On} R \pm, \Omega \pm *$ is the inverse of $\Omega^{ \pm}$, while it annihilates $\left.\left(R^{ \pm}\right)^{\perp}\right)$, and the $S$-operator is defined as an operator on $\mathscr{H}^{\prime}$ by

$$
S=\Omega^{+*} \Omega^{-} \text {. }
$$


$S$ is unitary if and only if

$$
R^{+}=R^{-}
$$

and then has the following interpretation: given any $\Phi=\left\{\varphi_{\alpha}\right\} \in \mathscr{H}^{\prime}$, there exists a state $\psi \in R^{-}$(namely $\left.\psi=\Omega^{-} \Phi\right)$ such that

$$
e^{L t} \psi \rightarrow \sum_{\alpha} e^{L_{\alpha} t} \varphi_{\alpha} \quad(t \rightarrow-\infty)
$$

in the sense of the norm. By (12), we also have $\psi \in R^{+}$, hence there exists a state $\Psi=\left\{\psi_{\alpha}\right\} \in \mathscr{H}^{\prime}$ (namely $\Psi=\Omega^{+*} \psi$ ) such that

$$
e^{L t} \psi \rightarrow \sum_{\alpha} e^{L_{\alpha} t} \psi_{\alpha} \quad(t \rightarrow+\infty),
$$

and the relation between the asymptotic behaviour in the past and the asymptotic behaviour in the future is precisely given by $S$ :

$$
\Psi=S \Phi .
$$

In the next section we shall prove not only (12) but

$$
R^{+}=R^{-}=\mathscr{H}
$$

(Asymptotic completeness), which says that for any $\psi \in \mathscr{H}, \exp (L t) \psi$ has the asymptotic behaviour (13) and (14) for $t \rightarrow \mp \infty$.

\section{Asymptotic Completeness}

If the $N$-particle system possesses bound states, there is a channel $\alpha=0=$ trivial partition into one fragment, for which $D_{0}=R_{0}^{ \pm}=\mathscr{H}^{B}$. So far there was no reason to treat this channel differently, but from now on we shall distinguish between bound states $\in \mathscr{H}^{B}$ and scattering states (in the proper sense) $\in S^{ \pm}$, where

$$
S^{ \pm} \bigoplus_{\alpha \neq 0} R_{\alpha}^{ \pm}
$$

The assertion (15) then takes the following form:

Theorem 3. If the potentials satisfy $(A, B, C)$, then

$$
\mathscr{H}^{B} \oplus S^{ \pm}=\mathscr{H} \text {. }
$$

In the remainder of this section, we shall prove (16) for $S^{+}$; the statement for $S^{-}$then follows by time-inversion. Proceeding by induction in $N$, we assume $N \geqq 2$ and that (16) holds for all systems with less than $N$ particles, which is obvious for $N=2$.

First, we reformulate the induction hypothesis. Let $D$ label all partitions $\left(C_{1}, C_{2}\right)$ of $(1 \ldots N)$ into two non-empty subsets $C_{1}, C_{2}$, and let $H_{D}=H$ minus all interactions linking $C_{1}$ and $C_{2}$, and $L_{D}=$ Liouville operator generated by $H_{D}$. Since each of the subsystems $C_{1}$ and $C_{2}$ is asymptotically complete, the same is true for the composite system $\left(C_{1}, C_{2}\right)$ characterized by $H_{D}$. This means that, for any $\psi \in \mathscr{H}, \exp \left(L_{D} t\right) \psi$ behaves for $t \rightarrow+\infty$ like a motion of non-interacting fragments of the system $H_{D}$, in the sense of (14) Since these fragments are also fragments of the fully interacting system characterized by $H$, we have. 
Lemma 2. Let $\psi^{+} \in \mathscr{H}$ and suppose that there exist states $\varphi_{D} \in \mathscr{H}$ such that, in the sense of the norm,

as $t \rightarrow+\infty$. Then $\psi^{+} \in S^{+}$.

$$
e^{L t} \psi^{+} \rightarrow \sum_{D} e^{L_{D} t} \varphi_{D}
$$

Given any $\psi \in \mathscr{H}$, we shall first use this lemma to split off a scattering state $\psi^{+}$from $\psi$ and then prove that the remainder $\left(\psi-\psi^{+}\right)$is a bound state.

For any $D=\left(C_{1}, C_{2}\right)$ we define

$$
\begin{gathered}
\varrho_{D}(z)=\min _{i \in C_{1}, k \in C_{2}}\left|x_{i}-x_{k}\right|, \\
A_{D}=\left\{z: \varrho_{D}\left(S^{t} z\right) \geqq a \text { for } 0 \leqq t<\infty\right\},
\end{gathered}
$$

where $a$ is the upper bound on the range of the forces. $A_{D}$ is closed and "absorbing", i.e. $S^{t} A_{D} \subset A_{D}$ for all $t>0$, and represents a region in phase space where the system is and remains separated into the two non-interacting subsystems $C_{1}, C_{2}$. The set of all $z$ whose trajectories $\left\{S^{t} z\right\}$ will reach one of these regions $A_{D}$ in the future is

$$
\sigma^{+}=\bigcup_{0 \leqq t<\infty} S^{-t} A, \text { with } A=\bigcup_{D} A_{D} .
$$

Since $A$ is absorbing, we have

$$
S^{t_{2}} A \subset S^{t_{1}} A \text { if } t_{1} \leqq t_{2},
$$

hence we can write $\sigma^{+}$as a countable union

$$
\sigma^{+}=\bigcup_{n=0,1,2 \ldots} S^{-n} A
$$

which shows that $\sigma^{+}$is measurable, since $A$ is closed. In view of lemma 2 and of the significance of $A$ we now expect

Lemma 3. For any $\psi \in \mathscr{H}$, let $\psi^{+}$be the part of $\psi$ on $\sigma^{+}$(i.e. the product of $\psi$ with the characteristic function of $\left.\sigma^{+}\right)$. Then $\psi^{+} \in S^{+}$.

Proof. Defining $\psi_{n}=$ part of $\psi$ on $S^{-n} A$, we have

$$
\lim _{n \rightarrow \infty}\left\|\psi_{n}-\psi^{+}\right\|=0
$$

by (18), (19) and the dominated convergence theorem. Since $S^{+}$is closed, it therefore suffices to prove $\psi_{n} \in S^{+}$for all $n$. By definition of $\psi_{n}$, supp $\exp (L n) \psi_{n} \subset A$, hence we can write

$$
e^{L n} \psi_{n}=\sum_{D} \varphi_{D}
$$

with supp $\varphi_{D} \subset \mathrm{A}_{D}$, which by the properties of $A_{D}$ implies

$$
e^{L t} \psi_{n}=\sum_{D} e^{L_{D}(t-n)} \varphi_{D}
$$

for $t \geqq n$. Hence $\psi_{n}$ satisfies the hypothesis of lemma 2 , which asserts that $\psi_{n} \in S^{+}$. 
It remains to prove $\psi-\psi^{+} \in \mathscr{H}^{B}$. To prepare this, we first note that the bound states can be characterized by their behaviour for $t>0$ only:

Lemma 4 [6]:

Let $\bar{N}^{+}(z)=\sup _{0 \leqq t<\infty} N\left(S^{t} z\right)$ and $B^{+}=\left\{z: \bar{N}^{+}(z)<\infty\right\}$. Then $\left(B^{+}-B\right)$ is of measure zero, hence

$$
\mathscr{H}^{B}=L^{2}\left(B^{+}\right) .
$$

Proof. Let $C$ be any compact $\subset \Gamma$ and

$C^{ \pm}$is compact and

$$
C^{ \pm}=\bigcap_{0 \leqq \pm t<\infty} S^{-t} C \text {. }
$$

$$
S^{t} C^{+}=\bigcap_{-t \leqq \tau<\infty} S^{-\tau} C
$$

is shrinking as $t$ increases (i.e. $S^{t_{1}} C^{+} \subset S^{t_{2}} C^{+}$if $t_{2} \leqq t_{1}$ ), but of constant measure $\mu\left(C^{+}\right)$. Therefore

$\mu\left(C^{+}\right)=\mu \quad\left(\bigcap_{0 \leqq t<\infty} S^{-t} C^{+}\right)=\mu \quad\left(\bigcap_{-\infty<t<+\infty} S^{-t} C\right)=\mu\left(C^{+} \cap C^{-}\right)$,

i.e. almost all trajectories $\left\{S^{t} z\right\}$ which stay in $C$ for $t>0$ also stay there for $t<0$ ("capture is a process of zero probability"). We now apply this to the sequence of compacts

$$
C_{n}=\{z: N(z) \leqq n, \quad H(z) \leqq n\},
$$

$n=1,2, \ldots$ Then we have, by $(20)$

$$
\mu\left[C_{n}^{+}-\left(C_{n}^{+} \cap C_{n}^{-}\right)\right]=0
$$

and also, by the definition of $B$ and $B^{+}$,

Therefore,

$$
B^{+}=\bigcup_{n} C_{n}^{+}, \quad B=\bigcup_{n}\left(C_{n}^{+} \cap C_{n}^{-}\right) .
$$

is of measure zero.

$$
B^{+}-B \subset \bigcup_{n}\left[C_{n}^{+}-\left(C_{n}^{+} \cap C_{n}^{-}\right)\right]
$$

By lemma 4 it now suffices to prove $\psi-\psi^{+} \in L^{2}\left(B^{+}\right)$or, in terms of phase space, that the complement of $\sigma^{+}$is contained in $B^{+}$. This is achieved by

Lemma 5. For any $z_{0} \in \Gamma$ there exists $C \subset \Gamma$ such that

(a) $z_{0} \in C$,

(b) the projection of $C$ onto configuration space is compact,

(c) if the trajectory $\left\{S^{t} z_{0}\right\}$ leaves $C$ as $t$ increases, it reaches $A$.

By (c) and by the definition of $\sigma^{+}, z_{0} \notin \sigma^{+}$implies $S^{t} z_{0} \in C$ for all $t>0$, hence $z_{0} \in B^{+}$by $(\mathrm{b})$.

Proof. To construct $C$, we define for any $D=\left(C_{1}, C_{2}\right)$

$d_{D}(z)=$ distance between the $\mathrm{CM}$ of $C_{1}$ and the $\mathrm{CM}$ of $C_{2}$, in the state $z$, $\bar{d}(z)=\max _{D} d_{D}(z)$

and we shall call a decomposition $D$ maximal (for a given $z$ ) if $d_{D}(z)=\bar{d}(z)$. With $\varrho_{D}$ defined by (17) we then have 
Proposition 1. There exists a constant $\lambda$ depending only on the masses, with $0<\lambda<1$, such that $d_{D}(z) \geqq \lambda \bar{d}(z)$ implies $\varrho_{D}(z) \geqq(1-\lambda) \bar{d}(z)$.

The proof will be given at the end of this section. The set $C$ of lemma 5 is now defined by

$$
C=\{z:(1-\lambda) \bar{d}(z) \leqq a\},
$$

where a is again an upper bound on the range of the forces, but chosen so large that $(1-\lambda) \bar{d}\left(z_{0}\right) \leqq a$. (a) is then satisfied by construction. For (b), consider the decomposition $D=\left(C_{1}, C_{2}\right)$ where $C_{1}$ consists of particle $i$ only. Then it is evident that $z \in C$ implies

$$
\left|x_{i}\right|<d_{D}(z) \leqq \bar{d}(z) \leqq a(1-\lambda)^{-1}
$$

for all $i$, which proves (b). The proof of (c) is obtained in several steps (propositions 2-4 below), based on proposition 1 and on the fact that the distance between two freely moving particles is a convex function of $t(f(t)$ is called convex in the interval $r \leqq t \leqq s$ if, in any subinterval $t_{1} \leqq t \leqq t_{2}, f(t) \leqq$ linear interpolation of $f(t)$ between $t_{1}$ and $\left.t_{2}\right)$. We study the functions $\bar{d}(t)$ and $d_{D}(t)$ along the trajectory $\left\{S t_{z_{0}}\right\}$ :

Proposition 2. There exists a constant $v<\infty$, independent of $D$ and $t$, such that

and therefore, for all $t, t_{0}$,

$$
\left|\dot{d_{D}}(t)\right| \leqq v
$$

$$
\left|\bar{d}(t)-\bar{d}\left(t_{0}\right)\right| \leqq v\left|t-t_{0}\right| .
$$

Proof. Let $M_{1}, M_{2}$ be the masses of the subsystems $C_{1}, C_{2}$ in the decomposition $D$. Then

$$
\frac{M_{1} M_{2}}{2\left(M_{1}+M_{2}\right)} \dot{d}_{D}^{2} \leqq T \leqq H\left(z_{0}\right)-b
$$

where $T$ is the kinetic energy and $b$ a lower bound on $V\left(x_{1} \ldots x_{N}\right)$. Hence (21) is satisfied for

$$
v=\left[\frac{2 M}{m(M-m)}\left(H\left(z_{0}\right)-b\right)\right]^{1 / 2},
$$

with $M=$ total mass of the system, $m=$ smallest particle-mass.

Proposition 3. $\bar{d}(t)$ is convex in any interval $I: r \leqq t \leqq s$ in which $(1-\lambda) \bar{d}(t) \geqq a$.

Corollary. $\dot{\vec{d}}(t) \equiv$ left derivative of $\bar{d}(t)$ exists for $r<t \leqq s$, is nondecreasing as $t$ increases, and satisfies $\dot{\vec{d}}(t) \leqq v$.

Proof. Let $t_{0} \in I$ and let $D$ be maximal at time $t_{0}$. Then, by proposition 2,

$$
d_{D}(t)-\bar{d}(t) \geqq a-2 v\left|t-t_{0}\right| \geqq 0
$$

for $\left|\ell-t_{0}\right| \leqq \varepsilon \equiv a / 2 v$. In the interval

$$
I \cap\left[t_{0}-\varepsilon, t_{0}+\varepsilon\right]
$$


we then have $\varrho_{D}(t) \geqq a$, by proposition 1 , i.e. no interaction between $C_{1}$ and $C_{2}$, which implies that $d_{D}(t)$ is convex. Therefore, for any $t_{0} \in I$, there exists an interval (22) in which $\bar{d}(t)$ is bounded below by a convex function taking the same value at $t=t_{0}$. Since $\varepsilon$ is independent of $t_{0}$, it follows that $\bar{d}(t)$ is convex in $I$.

We now assume that the trajectory $\left\{S^{t} z_{0}\right\}$ leaves $C$ as $t$ increases and prove that it reaches $A$. By assumption,

$$
(1-\lambda) \bar{d}\left(t_{1}\right)>a
$$

for some $t_{1}>0$ and also $\dot{\bar{d}}\left(t_{1}\right)>0$, since otherwise the inequality (23) would persist, by convexity, for all $t<t_{1}$, in contradiction to $(1-\lambda) \bar{d}(0) \leqq a$. Therefore $\bar{d}(t)$ is convex and strictly increasing for all $t \geqq t_{1}$, which implies

$$
(1-\lambda)^{-1} a \leqq \bar{d}(t) \rightarrow \infty
$$

and also, by the corollary of proposition 3 ,

$$
\begin{aligned}
& \dot{\bar{d}}(t) \rightarrow \dot{\bar{d}}(\infty) \leqq v, \\
& \dot{\bar{d}}(t) \leqq \dot{d}(\infty),
\end{aligned}
$$

for $t_{1} \leqq t \rightarrow \infty$.

Proposition 4. Let $t_{2} \geqq t_{1}$ be so large that $\dot{\bar{d}}\left(t_{2}\right)>\lambda \dot{\bar{d}}(\infty)$, and let $D$ be maximal at time $t_{2}$. Then $\varrho_{D}(t)>a$ for all $t \geqq t_{2}$, i.e. $S^{t_{2}} z_{0} \in A$.

Proof. Assume the contrary, i.e. $\varrho_{D}(t) \leqq a$ for some $t>t_{2}$. Then, by proposition $1, d_{D}(t) \leqq \lambda \vec{d}(t)$. Let $t_{3}$ be the smallest time $\geqq t_{2}$ for which $d_{D}\left(t_{3}\right)=\lambda \bar{d}\left(t_{3}\right)$. Then

$$
\dot{d}_{D}\left(t_{3}\right) \leqq \lambda \dot{\bar{d}}\left(t_{3}\right)
$$

since $d_{D}(t) \geqq \lambda \bar{d}(t)$ for $t_{2} \leqq t \leqq t_{3}$. For the same reason, $\varrho_{D}(t) \geqq a$ in this interval, by proposition 1 , so that $d_{D}(t)$ is convex and therefore

$$
\dot{d_{D}}\left(t_{2}\right) \leqq \dot{d}_{D}\left(t_{3}\right) \text {. }
$$

Finally, since $D$ is maximal at time $t_{2}$, we have $\bar{d}\left(t_{2}\right)=d_{D}\left(t_{2}\right)$ and $\bar{d}(t) \geqq d_{D}(t)$ for $t \leqq t_{2}$, which implies

$$
\dot{\bar{d}}\left(t_{2}\right) \leqq \dot{d}_{D}\left(t_{2}\right)
$$

Combining (25), (26), (27) we find

$$
\dot{\bar{d}}\left(t_{3}\right) \geqq \frac{1}{\lambda} \dot{\bar{d}}\left(t_{2}\right)>\dot{\bar{d}}(\infty),
$$

in contradiction to (24).

Proof of Proposition 1. We consider a fixed configuration $\left(x_{1} \ldots x_{N}\right)$ and a partition $D=\left(C_{1}, C_{2}\right)$. Notation: $M_{i}=$ mass of $C_{i}, M=$ total mass of the system, $m=$ smallest particle-mass, $S_{i}=\mathrm{CM}$ of $C_{i}$. 
In $R^{3}$ we choose a $\xi$-axis with origin $S_{1}$ and with the positive part through $S_{2}$. Let $\xi_{1}$ be the $\xi$-coordinate of a particle of mass $m_{1}$ belonging to $C_{1}$. We now consider the decomposition $D^{\prime}=\left(C_{1}^{\prime}, C_{2}^{\prime}\right)$ obtained from $D$ by transferring this particle from $C_{1}$ to $C_{2}$. The $\xi$-coordinates of the $\mathrm{CM} S_{i}^{\prime}$ of $C_{i}^{\prime}$ are then

$$
\begin{array}{ll}
S_{1}^{\prime}: & -\xi_{1} m_{1}\left(M_{1}-m_{1}\right)^{-1} \\
S_{2}^{\prime}: & \left(d_{D} M_{2}+\xi_{1} m_{1}\right)\left(M_{2}+m_{1}\right)^{-1},
\end{array}
$$

and from dist $\left(S_{1}^{\prime}, S_{2}^{\prime}\right) \leqq \bar{d}$ we obtain

or

$$
\bar{d} \geqq d_{I} M_{2}\left(M_{2}+m_{1}\right)^{-1}+\xi_{1} m_{1} M\left[\left(M_{1}-m_{1}\right)\left(M_{2}+m_{1}\right)\right]^{-1},
$$

$$
\xi_{1} \leqq\left(M_{1}-m_{1}\right)\left(M_{2}+m_{1}\right)\left(m_{1} M\right)^{-1}\left[\bar{d}-M_{2}\left(M_{2}+m_{1}\right)^{-1} d_{D}\right] .
$$

Note that this also holds if $C_{1}$ consists of only one particle (then $\xi_{1}=0$ and $M_{1}=m_{1}$ ) in spite of the fact that the derivation does not. Similarly, considering a particle of mass $m_{2}$ belonging to $C_{2}$, we obtain for its $\xi$-coordinate:

$\xi_{2} \geqq d_{D}-\left(M_{2}-m_{2}\right)\left(M_{1}+m_{2}\right)\left(m_{2} M\right)^{-1}\left[\bar{d}-M_{1}\left(M_{1}+m_{2}\right)^{-1} d_{D}\right]$.

In particular, (28) and (29) hold for the two particles with distance $\varrho_{D}$, hence we obtain

$$
\begin{aligned}
\varrho_{D} \geqq & \xi_{2}-\xi_{1} \\
\geqq & -\bar{d}\left(m_{1} m_{2} M\right)^{-1}\left[m_{1}\left(M_{1}+m_{2}\right)\left(M_{2}-m_{2}\right)\right. \\
& \left.+m_{2}\left(M_{2}+m_{1}\right)\left(M_{1}-m_{1}\right)\right] \\
& +d_{D}\left\{1+\left(m_{1} m_{2} M\right)^{-1}\left[m_{1} M_{1}\left(M_{2}-m_{2}\right)+m_{2} M_{2}\left(M_{1}-m_{1}\right)\right]\right\} .
\end{aligned}
$$

Dividing by $\{\ldots\}$, one finds after some manipulations and by using $\{\ldots\} \geqq 1$ :

with

$$
d_{D} \leqq \varrho_{D}+\vec{d} \alpha\left(\alpha+m_{1}+m_{2}\right)^{-1}
$$

$$
\alpha=m_{2}^{-1}\left(M_{2}-m_{2}\right)\left(M_{1}+m_{2}\right)+m_{1}^{-1}\left(M_{1}-m_{1}\right)\left(M_{2}+m_{1}\right) .
$$

Since $a+b=M$ implies $a b \leqq M^{2} / 4$, we have $\alpha \leqq M^{2} / 2 m$ and therefore which yields

$$
\alpha\left(\alpha+m_{1}+m_{2}\right)^{-1} \leqq M^{2}\left(M^{2}+4 m^{2}\right)^{-1},
$$

$$
d_{D} \leqq \varrho_{D}+\bar{d} M^{2}\left(M^{2}+4 m^{2}\right)^{-1} .
$$

This proves the assertion of proposition 1 for

$$
\lambda=\left(M^{2}+2 m^{2}\right)\left(M^{2}+4 m^{2}\right)^{-1} .
$$

Remark: The proof actually shows that $d_{D} \geqq \lambda \bar{d}$ implies not only $\varrho_{D} \geqq(1-\lambda) \bar{d}$ but

$$
\min _{i \in C_{2}, k \in C_{1}}\left(\xi_{i}-\xi_{k}\right) \geqq(1-\lambda) \bar{d}
$$


i.e. that there exist two parallel planes (orthogonal to the $\xi$-axis) with distance $\geqq(1-\lambda) \bar{d}$ which both separate $C_{1}$ and $C_{2}$. This shows, for example, that the convexity argument also works for repulsive forces of infinite range.

\section{Infinite-range Forces}

Let us attempt to prove the existence of scattering states as in quantum mechanics [7]. Differentiating $\exp (-L t) \exp \left(L_{\alpha} t\right) \psi_{\alpha}$ with respect to $t$ and integrating again from 0 to $t$ we formally obtain

with

$$
e^{-L t} e^{L_{\alpha} t} \psi_{\alpha}=\psi_{\alpha}-\int_{0}^{t} d \tau e^{-L \tau}\left(L-L_{\alpha}\right) e^{L_{\alpha} \tau} \psi_{\alpha},
$$

$$
L-L_{\alpha}=\Sigma^{\prime} \operatorname{grad} V_{i k}\left(\frac{\partial}{\partial p_{i}}-\frac{\partial}{\partial p_{k}}\right)
$$

where the sum is restricted to the pairs linking different fragments of channel $\alpha$. One then tries to prove the convergence of the left-hand side of (30) (for a dense set of states $\psi_{\alpha} \in D_{\alpha}$ ) by showing that the integral on the right converges absolutely for $t= \pm \infty$, i.e. that

$$
\int_{0}^{ \pm} d t\left\|\left(L-L_{\alpha}\right) e^{L_{\alpha} t} \psi_{\alpha}\right\|<\infty .
$$

Recalling (31) and the explicit form (8) of $\exp \left(L_{\alpha} t\right) \psi_{\alpha}$ we see that we need a dense set of differentiable bound states for each fragment (dense in $\left.\mathscr{H}^{B}\right)$. But such a set exists only if the boundary $(B-\operatorname{int} B)$ of $B$ is of measure zero. Actually we shall assume slightly more, namely that

$$
\mu\left(B-\bigcup_{n} \operatorname{int} B^{n}\right)=0 .
$$

Technically, this means that we can approximate bound states by smooth, compact bound states. Physically, (33) is a stability assumption, for $z \in U$ int $B^{n}$ means that there exists $n<\infty$ and $\varepsilon>0$ such that $\bar{N}\left(z^{\prime}\right) \leqq n$ if $\left|z^{\prime}-z\right|<\varepsilon$, i.e. that, for sufficiently small perturbations of the initial data $z$, all perturbed orbits stay in some compact of configuration space. (33) then requires that almost all bounded orbits are stable in this sense. Whether this is true or not seems at present to be open.

We now take $\psi_{\alpha}$ of the same form as in the proof of theorem 1 , but with the additional property that $F, G, \varphi_{1} \ldots \varphi_{n}$ are once continuously differentiable. By (33), these states still span a dense set in $D_{\alpha}$, and the formal derivation of (30) is justified by lemma 1. In order to estimate the integrand in (32), we express the differential operators in (31) as linear combinations of $\partial / \partial P_{k}$ and $\partial / \partial \pi_{k}$, where $P_{k}=$ total momentum of the fragment $F_{k}, \pi_{k}=$ internal momenta in $F_{k}$, and define

$$
f_{i k}(r)=\sup _{|x| \geqq r}\left|\operatorname{grad} V_{i k}(x)\right|
$$


There are two essentially different types of contributions to the integrand in (32):

a) Contribution from grad $V_{i{ }_{i}} \partial / \partial P_{j}$

$$
\begin{aligned}
& \| \operatorname{grad} V_{i k}\left[-\frac{t}{M_{j}} \frac{\partial F}{\partial X_{j}}\left(X_{1}-\frac{P_{1}}{M_{1}} t, \ldots, X_{n}-\frac{P_{n}}{M_{n}} t\right) G\left(P_{1} \ldots P_{n}\right)\right. \\
& \left.\quad+F\left(X_{1}-\frac{P_{1}}{M_{1}} t, \ldots, X_{n}-\frac{P_{n}}{M_{n}} t\right) \frac{\partial G}{\partial P_{j}}\left(P_{1} \ldots P_{n}\right)\right] \prod_{k=1}^{n}\left(e^{l_{n} t} \varphi_{k}\right)\left(z_{k}\right) \| \\
& \quad \leqq \text { const. }(1+|t|) f_{i k}(\sigma|t|-3 R),
\end{aligned}
$$

which is integrable over $-\infty<t<+\infty$ if

$$
\int_{0}^{\infty} d r(1+r) f_{i k}(r)<\infty,
$$

i.e. if the forces decrease faster than the Coulomb force at infinity.

$$
\text { b) Contribution from grad } V_{i k} \partial / \partial \pi_{j}
$$

$$
\begin{aligned}
& \| \operatorname{grad} V_{i k} F\left(X_{1}-\frac{P_{1}}{M_{1}} t, \ldots, X_{n}-\frac{P_{n}}{M_{n}} t\right) G\left(P_{1} \ldots P_{n}\right) \\
& \cdot\left(\frac{\partial}{\partial \pi_{j}} e^{l_{j} t} \varphi_{j}\right)\left(z_{j}\right) \prod_{k \neq j}\left(e^{l_{k} t} \varphi_{k}\right)\left(z_{k}\right) \| \leqq \text { const. } f_{i k}(\sigma|t|-3 R)\left\|\frac{\partial}{\partial \pi_{j}} e^{l_{j} t} \varphi_{j}\right\| .
\end{aligned}
$$

Since, in general, we only have the exponential estimate (4) for the last norm and since $\sigma$ is arbitrarily small, we need

$$
\int_{0}^{\infty} d r e^{\alpha r} f_{i k}(r)<\infty \quad \text { for any } \alpha>0
$$

in order to assert that the contribution of this term to the integral (32) is finite. Note, however, that if a linear estimate

$$
\left\|\frac{\partial}{\partial \pi_{j}} e^{l_{j} t} \varphi_{3}\right\| \leqq \text { const. }(1+|t|)
$$

is available, then again condition (34) is sufficient.

Having exhibited the difficulties arising from infinite-range forces, we now discuss some simple cases in which these problems do not occur or can be handled:

\section{No composite fragments}

For the channel $\alpha=$ decomposition of $(1 \ldots N)$ into $N$ single particles, no information on bound states is needed and the wave operators $\Omega_{\alpha}^{ \pm}$exist as strong limits on $D_{\alpha}$ if only (34) is satisfied. In particular, this suffices for $N=2$, and for arbitrary $N$ if the forces are repulsive. (To see that no bound states exist for repulsive forces, note that $N(t)=N\left(S^{t} z\right)$ as defined by (5) satisfies

$$
\frac{d^{2}}{d t^{2}} N^{2}(t)=2 T-\sum_{i<k}\left(x_{i}-x_{k}\right) \operatorname{grad} V_{i k} \geqq 2 T \geqq 0,
$$


$T$ being the kinetic energy. Hence $N^{2}(t)$ is convex and therefore bounded only if it is constant, which implies $T=0$. Consequently, $B$ is contained in the null-set $p_{1}=p_{2}=\cdots=p_{N}=0$.)

\section{Fragments consisting of two particles}

For two-particle systems with a spherically symmetric potential, (33) is easily verified and (35) can be proved as indicated below. In the case of central forces (34) is therefore sufficient to assert the existence of $\Omega_{\alpha}^{ \pm}$for all channels in which only single particles and (or) two-body bound states are present as $t \rightarrow \pm \infty$. In particular, this should allow a complete scattering theory for the case $N=3$ with central forces decreasing faster than the Coulomb force.

For simplicity, we shall sketch the proof of (35) for $N=2$ in one dimension only, but the same argument also works in the 3 -dimensional case after separation of variables. In addition to $(A, B)$ we assume that $V(x)$ takes only a finite number of values $E_{1} \ldots E_{r}$ on the set $\{x: d V / d x(x)=0\}$. Let $O$ be the (open) part of $B$ in the complement of the energy-shells $S_{n}=\left\{z: H(z)=E_{n}\right\}, n=1 \ldots r$. For each $z \in O$, the motion $S^{t} z$ is periodic with a period

$$
T(z)=\int_{a(z)}^{b(z)} d s\left[2 \mu^{-1}(H(z)-V(s))\right]^{-1 / 2},
$$

( $\mu=$ reduced mass, $a, b=$ turning points) which is once continuously differentiable with respect to $z$ (but may diverge as $z$ approaches one of the energy-shells $S_{n}$ ). Therefore we can apply

Lemma 6. Let $O \subset T$ be open and suppose that there exists a function $T(z)>0$ and once continously differentiable on $O$ such that

$$
S^{T(z)} z=z
$$

for all $z \in O$. Then, for any compact $C \subset O$, there exists a constant $k(C)<\infty$ such that (matrix norm)

for all $z \in C$.

$$
\left|\frac{d}{d z} S^{t} z\right| \leqq k(C)(1+|t|)
$$

As a consequence, we see that (35) is satisfied in our 1-dimensional example for the set $C_{0}^{1}(O)$ of compact bound states which is dense in $\mathscr{H}^{B}$.

Proof. Differentiating

$$
S^{t} z=S^{t \pm T(z)} z
$$

with respect to $z$ and using the equations of motion we obtain

$$
A_{i j}(t)=A_{i j}(t \pm T) \pm \varepsilon_{i k} \frac{\partial H}{\partial z_{k}}(t \pm T) \frac{\partial T}{\partial z_{j}},
$$


where $A_{i j}(t)=\partial\left(S^{t} z\right)_{i} / \partial z_{j}$. Since $C$ is compact, there exist constants $b_{1} \ldots b_{4}$ such that, uniformly in $z \in C$,

hence

$$
\begin{gathered}
\left|\frac{d H}{d z}(t)\right| \leqq b_{1}<\infty \quad \text { for all } t \\
\left|\frac{d T}{d z}\right| \leqq b_{2}<\infty \\
0<b_{3} \leqq T(z) \leqq b_{4}<\infty
\end{gathered}
$$

$$
|A(t)| \leqq|A(t \pm T)|+b_{1} b_{2},
$$

where | | denotes the Euclidean norm in (36), (37) and the corresponding matrix-norm in (38). For $t \lessgtr 0$ we iterate the appropriate inequality (38) $n$ times until $|t \pm n T| \leqq T$. Then $|n| \leqq|t| / b_{3}$ and $|A(t \pm n T)| \leqq \exp \left(K b_{4}\right)$ by (3), hence we obtain the desired result:

$$
|A(t)| \leqq e^{K b_{1}}+|t| \frac{b_{1} b_{2}}{b_{3}}
$$

Note that $b_{2}=0$ if $T(z)=$ constant (harmonic oscillator), then (39) reduces to a $t$-independent estimate.

So far, we have discussed the problem of extending theorem 1 to infinite-range forces. If this is possible, then the $S$-matrix can be constructed as before, since theorem 2 is of a purely kinematical nature (no assumptions on the forces are needed in its proof). However, the proof of asymptotic completeness was again based strongly on the finite range of the forces, and we do not know at present if theorem 3 holds in the cases where theorem 1 can be proved. An exception is the case $N=2$, where it is sufficient to require that the forces and their first derivatives vanish with a higher power of $1 / r$ for $r \rightarrow \infty$ than the corresponding expressions for the Coulomb force.

\section{Scattering in Terms of Phase-Space}

Our description of collisions in terms of $\mathscr{H}=L^{2}(T)$ was motivated by the analogy to quantum mechanics, but everything can of course be transcribed into the language of phase-space. In particular, we expect that the wave-operators and the $S$-operator are induced by transformations in phase-space, just as $\exp (L t)$ was induced by $S^{t}$. We shall sketch this briefly for the case of finite-range forces.

Instead of theorem 1 we have, in the notation of section 3 :

Theorem I. Let $d_{\alpha}$ be the subset of $\delta_{\alpha}$ where $P_{i} / M_{i} \neq P_{k} / M_{k}(i \neq k)$. Then the limits

$$
z_{\alpha}^{ \pm}=\lim _{t \rightarrow \pm \infty} S^{-t} S_{\alpha}^{t} z
$$


exist for all $z \in d_{\alpha}$ and define the mappings $\omega_{\alpha}^{ \pm}: z \rightarrow z_{\alpha}^{ \pm}$from $d_{\alpha}$ into $\Gamma$ with the following properties:

(a) $\omega_{\alpha}^{ \pm}$is one-to-one and preserves measurability and measure. In particular, the ranges $Q_{\alpha}^{ \pm}$of $\omega_{\alpha}^{ \pm}$are measurable.

(b) $S^{t} \omega_{\alpha}^{ \pm} z=\omega_{\alpha}^{ \pm} S_{\alpha}^{t} z$ for all $t$ and all $z \in d_{\alpha}$. Hence $\varrho_{\alpha}^{ \pm}$is invariant under the group $S^{t}$.

(c) For any $\psi_{\alpha} \in D_{\alpha}$ we have almost everywhere

$$
\left(\Omega_{\alpha}^{ \pm} \psi_{\alpha}\right)(z)=\left\{\begin{array}{cll}
\psi\left[\left(\omega_{\alpha}^{ \pm}\right)^{-1} z\right] & \text { if } z \in Q_{\alpha}^{ \pm} \\
0 & \text { if } z \notin Q_{\alpha}^{ \pm}
\end{array} .\right.
$$

(Note that $\mu\left(\delta_{\alpha}-d_{\alpha}\right)=0$ ). Hence

Proof. We have

$$
R_{\alpha}^{ \pm}=L^{2}\left(Q_{\alpha}^{ \pm}\right)
$$

with

$$
d_{\alpha}=\bigcup_{m=1,2, \ldots} d_{\alpha}^{m}
$$

$$
d_{\alpha}^{m}=I_{\alpha}^{m} \otimes B_{1}^{m} \otimes \cdots \otimes B_{n}^{m},
$$

where the $B_{k}^{m}$ are defined by $(6)$ for each fragmet $F_{k}$, and where

$$
\Gamma_{\alpha}^{m}=\left\{\left(X_{1} \ldots X_{n}, P_{1} \ldots P_{n}\right):\left|\frac{P_{i}}{M_{i}}-\frac{P_{k}}{M_{k}}\right|>\frac{1}{m} \quad(i \neq k)\right\} .
$$

By the inequalities used in the proof of theorem 1 one then finds that, for each $m$, there exists a finite time $T(m)$ such that, on $d_{\alpha}^{m}, \omega_{\alpha}^{ \pm}$coincides with the canonical transformation

$$
S^{-T(m)} S_{\alpha}^{T(m)} \text {. }
$$

The rest of the proof is obvious.

Instead of theorem 2 we have, with the same proof,

Theorem II.

$$
\varrho_{\alpha}^{ \pm} \cap \varrho_{\beta}^{ \pm}=\text {empty if } \alpha \neq \beta \text {. }
$$

The definition of $\mathscr{H}^{\prime}, \Omega^{ \pm}$and $S$ is parallelled by the following construction: we have $\mathscr{H}^{\prime}=L^{2}\left(\Gamma^{\prime}\right)$, where

$$
\Gamma^{\prime}=\left\{(z, \alpha): z \in \delta_{\alpha}\right\}
$$

is the disjoint union of the sets $\delta_{\alpha}$, fitted with topology and measure already defined for its components. Just as $\mathscr{H}^{\prime}$ cannot be viewed as a subspace of $\mathscr{H}, \Gamma^{\prime}$ is not a subset of $\Gamma$ since in $\Gamma$ the sets $\delta_{\alpha}$ overlap. We call $\Gamma^{\prime}$ the "phase-space of asymptotes", since each element $(z, \alpha) \in \Gamma$ ' defines an "asymptote" $\left\{S_{\alpha}^{t} z\right\}$ representing a motion of noninteracting bound fragments. We then define the mapping $\omega^{ \pm}$from $\Gamma^{\prime}$ into $\Gamma$ by

$$
\omega^{ \pm}(z, \alpha)=\omega_{\alpha}^{ \pm} z
$$

its domain being the disjoint union of the domains $d_{\alpha}$, and its range

$$
\varrho^{ \pm}=\bigcup_{\alpha} \varrho_{\alpha}^{ \pm}
$$


Again, $\omega^{ \pm}$is one-to-one, measure preserving, and induces $\Omega^{ \pm}$by

$$
\left(\Omega^{ \pm} \Phi\right)(z)=\left\{\begin{array}{cl}
\Phi\left[\left(\omega^{ \pm}\right)^{-1} z\right] & \text { if } z \in \varrho^{ \pm} \\
0 & \text { if } z \notin \varrho^{ \pm}
\end{array}\right.
$$

(a.e.) for any $\Phi \in L^{2}\left(\Gamma^{\prime}\right)$. Since $R^{ \pm}=L^{2}\left(\varrho^{ \pm}\right)$, theorem 3 now reads :

Theorem III.

$$
\mu\left(\Gamma-\varrho^{ \pm}\right)=0 \text {. }
$$

Finally, we consider the mapping

$$
s=\left(\omega^{+}\right)^{-1} \omega^{-}
$$

from $\Gamma^{\prime}$ into $\Gamma^{\prime}$. Its domain and range are $\left(\omega^{-}\right)^{-1}\left(\varrho^{+} \cap \varrho^{-}\right)$and $\left(\omega^{+}\right)^{-1}\left(\varrho^{+} \cap \varrho^{-}\right)$, respectively, and for any $z \in\left(\varrho^{+} \cap \varrho^{-}\right), s$ maps the asymptote of the trajectory $\left\{S^{t} z\right\}$ for $t \rightarrow-\infty$ onto the asymptote for $t \rightarrow+\infty$. Furthermore, $s$ is one-to-one and measure-preserving, and by virtue of theorem III, both the domain and the range of $s$ have complements of measure zero in $\Gamma^{\prime}$. In terms of $s$, the $S$-operator is given by

$$
(S \Phi)(z, \alpha)=\Phi\left[s^{-1}(z, \alpha)\right]
$$

for almost all $(z, \alpha) \in \Gamma^{\prime}$.

Remark: If (33) holds for all fragments of the system we modify the definition of $d_{\alpha}$ by taking

where now

$$
d_{\alpha}=\bigcup_{m=1,2, \ldots} d_{\alpha}^{m}
$$

$$
d_{\alpha}^{m}=\Gamma_{\alpha}^{m} \otimes \operatorname{int} B_{1}^{m} \otimes \cdots \otimes \operatorname{int} B_{n}^{m} .
$$

This $d_{\alpha}$ then differs from the previous one only by a set of measure zero, and all the statements of this section remain valid. In addition, however, $d_{\alpha}, \varrho_{\alpha}^{ \pm}$, and the domains and ranges of $\omega_{\alpha}^{ \pm}, \omega^{ \pm}$and $s$ are now open and the mappings $\omega_{\alpha}^{ \pm}, \omega^{ \pm}$and $s$ are canonical.

\section{References}

1. After this was written, we learned that J. M. Cook had already treated the case $N=2$ in the same spirit (see 1965 Cargèse Lectures in Theoretical Physics, edited by F. Lurcat. New York: Gordon \& Breach 1967).

2. Kato, T.: Trans. Am. Math. Soc. 70, 195 (1951).

3. Nelson, E.: Operator differential equations, Lemma 12.1, mimeographed lecture notes. Princeton University 1964.

4. Ruelle, D., unpublished.

5. Essentially we follow $J_{A \cup C H}$, J. M., Helv. Physica Acta 31, 661 (1958), but we prefer a different definition of the $S$-operator, due to Berfazr, F. A., L. D. Faddeev, and R. A. Mrncos, Proceedings of the Fourth All-Union Mathematical Conference, held in Leningrad 1961.

6. This is a classical result: see SregEL, C. L.: Vorlesungen über Himmelsmechanik, $\S 30$, Berlin, Göttingen, Heidelberg: Springer 1956. I am indebted to R. JosT for this remark.

7. For the quantum mechanical proof see HAck, M. N.: Nuovo Cimento 13, 231 (1959).

Dr. W. HuNZIKER

permanent address:

Seminar f. theor. Physik der ETH

Ch - 8044 Zürich, Hochstr. 60 